\section{Sindrome dos Ovários Policisticos, Sindrome Metabólica, Risco Cardiovascular e o Papel dos Agentes Sensibilizadores da Insulina}

\section{RESUMO}

A Síndrome dos Ovários Policísticos (SOP) afeta de 6 a 10\% das mulheres em idade reprodutiva. Resistência à insulina e hiperinsulinemia estão presentes em praticamente todas as pacientes com SOP e desempenham papel central no desenvolvimento tanto do hiperandrogenismo como da síndrome metabólica (SM). SM ocorre em aproximadamente $43 \%$ das pacientes com SOP, elevando em até sete vezes o risco de doença cardiovascular nestas pacientes. Vários marcadores séricos, funcionais e estruturais de disfunção endotelial e de aterosclerose subclínica foram descritos em pacientes com SOP, mesmo nas jovens e não-obesas. Entretanto, embora a SOP afete adversamente o perfil cardiovascular, estudos a longo prazo não demonstraram consistentemente aumento da mortalidade cardiovascular, a qual parece ser mais observada no período da pós-menopausa. Recentemente, os anticoncepcionais orais estão sendo substituídos pelos agentes sensibilizadores de insulina (metformina e glitazonas) no tratamento da SOP, devido aos seus efeitos sobre a resistência à insulina e o risco cardiovascular. (Ara Bras Endocrinol Metab 2006;50/2:281-290)

Descritores: Síndrome dos ovários policísticos; Síndrome metabólica; Resistência à insulina; Risco cardiovascular; Metformina; Glitazonas

\section{ABSTRACT}

Polycystic Ovary Syndrome, Metabolic Syndrome, Cardiovascular Risk and the Role of Insulin Sensitizing Agents.

The Polycystic Ovary Syndrome (PCOS) affects 6 to $10 \%$ of women of childbearing age. Insulin resistance and hyperinsulinemia are present in nearly all PCOS patients and play a central role in the development of both hyperandrogenism and metabolic syndrome (MS). MS occurs in approximately $43 \%$ of PCOS patients, raising the cardiovascular risk to up seven fold in these patients. Several serum, functional and structural markers of endothelial dysfunction and subclinical atherosclerosis were described in PCOS patients, even those young and non-obese. However, despite the fact that PCOS adversely affects the cardiovascular profile, long-term studies did not demonstrate a consistent raise in cardiovascular mortality, which seems to be more observed in the postmenopausal period. Recently, oral contraceptives are being substituted for insulin sensitizing agents (metformin and glitazones) in the PCOS treatment, due to their effects on insulin resistance and cardiovascular risk. (Arq Bras Endocrinol Metab 2006;50/2:281-290)

Keywords: Polycystic ovary syndrome; Metabolic syndrome; Insulin resistance; Cardiovascular risk; Metformin; Glitazones revisão

\author{
Regina do Carmo Silva \\ Dolores P. Pardini \\ Claudio E. Kater
}

Disciplina de Endocrinologia da Universidade Federal de São Paulo (UNIFESP/EPM), São Paulo, SP.

Recebido em 30/10/05

Aceito em 17/01/06 
A SÍNDROME DOS OVÁRIOS POLICÍSTICOS (SOP) é uma das desordens endocrinológicas mais freqüentes em mulheres na idade reprodutiva, com prevalência de 6 a 10\% (1). Estima-se que, no mundo todo, 105 milhões de mulheres entre 15 e 49 anos de idade (sendo 4 milhões americanas) apresentem a SOP, a qual é responsável por 72 a $82 \%$ das causas de hiperandrogenismo (2-4).

A SOP engloba um amplo espectro de sinais e sintomas de disfunção ovariana. Em 2003, o consenso de Rotterdam propôs que a SOP pode ser diagnosticada após a exclusão de outras causas de irregularidade menstrual e hiperandrogenismo (hiperprolactinemia, formas não-clássicas das hiperplasias adrenais congênitas, síndrome de Cushing, neoplasias secretoras de andrógenos, hipotireoidismo) e a presença de pelo menos dois dos seguintes critérios: oligo e/ou anovulação (cujas manifestações clínicas são a oligomenorréia ou amenorréia, o sangramento uterino disfuncional e a infertilidade), níveis elevados de andrógenos circulantes (hiperandrogenemia) e/ou manifestações clínicas do excesso androgênico (hiperandrogenismo, caracterizado por hirsutismo, acne e alopécia) e morfologia policística dos ovários (presença de 12 ou mais folículos, medindo 2 a $9 \mathrm{~mm}$ de diâmetro e/ou volume ovariano acima de $10 \mathrm{~cm}^{3}$ ) à ultra-sonografia (US) $(1,5)$.

A agregação familial sugere que a SOP é uma desordem complexa e multigênica. Variantes genômicas em genes relacionados à biossíntese, regulação e ação dos andrógenos (CYP17, CYP21, CYP11 $\alpha, 17 \beta$ HSD5, SHBG, receptor androgênico, 11 $\beta-H S D$ e $H 6 P D)$, à ação e à secreção da insulina (INSR, VNTR, IRS-1, IRS-2, CAPN10, PPAR $\gamma$, sistema IGF), à secreção e à ação das gonadotrofinas (folistatina) e à síntese e metabolismo do ácido retinóico, assim como genótipos pró-inflamatórios (variantes dos genes do $T N F-\alpha, I L-6)$ podem estar envolvidos na predisposição genética da SOP (1,6-10).

$\mathrm{Na}$ SOP, a biossíntese aumentada de andrógenos está relacionada ao aumento da expressão do gene CYP17 e à maior estabilidade do seu RNA mensageiro nas células da teca (8), além de uma anormalidade no eixo hipotálamo-hipófise-ovários, onde o aumento da freqüência de pulsos do hormônio hipotalâmico liberador de gonadotrofinas $(\mathrm{GnRH})$ favorece a transcrição da sub-unidade $\beta$ do hormônio luteinizante (LH) sobre aquela do hormônio folículo-estimulante (FSH). Não se sabe se essa freqüência aumentada de pulsos de GnRH se deve a uma alteração intrínseca do pulso gerador hipotalâmico ou se é secundária aos baixos níveis de progesterona resul- tantes dos infreqüentes eventos ovulatórios observados na SOP. O aumento relativo da secreção hipofisária de LH ocasiona aumento de produção de androstenediona pelas células da teca, a qual é convertida pela 17 $\beta$-hidroxiesteróide desidrogenase do tipo 5 (17 $\beta$ HSD) em testosterona ou aromatizada em estrona (El). Nas células da granulosa, estrona é convertida em estradiol (E2) (1).

Antecedentes de baixo peso ao nascer e pubarca precoce conferem risco aumentado para o aparecimento da SOP (11), cujos sintomas usualmente se iniciam na época da menarca. Início após a puberdade também pode ocorrer como resultado de modificadores ambientais, tais como ganho de peso e vida sedentária (1).

\section{PAPEL DA INSULINA NA SOP}

Pelo menos 50\% das mulheres com SOP são obesas e a maioria, senão todas, apresenta resistência à insulina (intrínseca à SOP e independente da obesidade) e hiperinsulinemia, as quais se caracterizam clinicamente pela presença de acanthosis nigricans $(1,12)$.

Vários dados confirmam a hipótese de que a resistência à insulina e a hiperinsulinemia exerçam um papel patogênico na SOP. A nível central, a insulina parece estar envolvida na secreção anormal do LH e, a nível periférico, promove a secreção ovariana de andrógenos, através do aumento da expressão do gene CYPl7 e da atividade do citocromo P450cl7, tendo ação sinérgica com o LH, tanto diretamente como através do estímulo da secreção do fator de crescimento símile à insulina 1 (IGF-1). A insulina diminui a síntese hepática de IGFBP-1 e da globulina ligadora dos hormônios sexuais (SHBG), aumentando os níveis de andrógenos livres. Além disso, também pode potencializar, in vivo, a produção androgênica adrenal estimulada pelo hormônio adrenocorticotrófico (ACTH) e aumentar o metabolismo do cortisol (aumento da atividade da $5 \alpha$-redutase do tipo 1 no fígado e na pele) $(1,13)$.

A insulina regula a esteroidogênese através da ação sobre seu próprio receptor, a qual é mediada por três vias principais: fosfatidil-inositol 3-quinase (efeitos metabólicos), MAPK (mitogen-activated protein kinase - efeitos mitogênicos) e proteína quinase C. Existe intercomunicação entre essas vias de sinalização da insulina, assim como entre as vias de sinalização da insulina e do LH (14). Recentemente, foi proposta a existência, na SOP, de uma hipersensibilidade das células da teca ovariana à ação da insulina, defeito este 
intrínseco e não secundário ao estímulo crônico pelo LH e que pode ocorrer na ausência de resistência à insulina global ou hiperinsulinemia. Também foi descrito um defeito seletivo da ação da insulina nas células da granulosa de pacientes com SOP (resistência no caminho metabólico associado ao aumento da atividade mitogênica) (14).

A associação de resistência à insulina, inflamação e hiperandrogenismo pode ser decorrente da ação de fatores ambientais (insulto intra-uterino, obesidade, sedentarismo, dieta inadequada), influenciados pela etnia, os quais agiriam sobre um fino balanço entre variantes genéticas protetoras e predisponentes da SOP, selecionadas durante a evolução (6).

\section{SOP COMO COMPONENTE DA SÍNDROME METABÓLICA}

Tanto as pacientes obesas como as não-obesas com SOP apresentam aumento da razão cintura-quadril e adipócitos de maior tamanho, quando comparadas às mulheres sem SOP, pareadas para o índice de massa corpórea (IMC) $(15,16)$. Sabe-se que o tecido adiposo visceral é responsável pela secreção de citocinas próinflamatórias, tais como interleucina 6 (IL-6) e fator de necrose tumoral a (TNF- $\alpha$ ), os quais participam na gênese da resistência à insulina (17). O TNF- $\alpha$ determina hiperfosforilação pós-transcripcional dos resíduos de serina tanto do IRS-1 como da enzima esteroidogênica $\mathrm{P} 450 \mathrm{cl} 7$, ocasionando, respectivamente, diminuição da atividade tirosina quinase do receptor da insulina (menor transdução do sinal) e aumento seletivo da atividade 17,20-liase da $\mathrm{P} 450 \mathrm{cl} 7(17,18)$.

Além disso, o aumento da geração de espécies reativas de oxigênio por células mononucleares, presentes no compartimento estromal vascular do tecido adiposo visceral das pacientes com $S O P$, em resposta à hiperglicemia fisiológica pós-prandial, pode desempenhar um papel importante no desenvolvimento do hiperandrogenismo e da resistência à insulina, através da ativação do NF- $\mathrm{NB}$ e do aumento da transcrição do gene do TNF- $\alpha$, caracterizando a SOP como um estado pró-inflamatório $(19,20)$.

A SOP é importante fator de risco para diabetes mellitus do tipo 2 (DM2) em adolescentes e mulheres na pré-menopausa. Cerca de $30 \%$ das mulheres com SOP apresentam tolerância à glicose diminuída e aproximadamente $10 \%$ delas têm DM2, taxas de prevalência muito maiores que as observadas em mulheres americanas normais, entre 20 e 44 anos de idade (7,8\% para tolerância à glicose diminuída e $1 \%$ para
DM2 não-diagnosticado) (21). Embora o risco de desenvolver tolerância à glicose diminuída ou DM2 aumente de acordo com o grau de obesidade ( $90 \%$ das mulheres diabéticas apresentam IMC acima de 30 $\mathrm{kg} / \mathrm{m}^{2}$ ), ele está presente também nas não-obesas e é maior nas pacientes com história familiar de DM2 $(21,22)$. As mulheres com SOP e tolerância à glicose diminuída apresentam risco de conversão para DM2 de $2 \%$ ao ano; aquelas com tolerância normal à glicose apresentam risco de conversão para intolerância de $16 \%$ ao ano. Isso enfatiza a necessidade da reavaliação periódica dessas pacientes com o teste oral de tolerância à glicose $(23)$.

Tem sido observado que os níveis de colesterol total e de LDL-colesterol estão aumentados nas pacientes obesas e não-obesas, mas apenas as obesas apresentam níveis de triglicérides aumentados. Nestas, os níveis de HDL-colesterol, embora inferiores a 50 $\mathrm{mg} / \mathrm{dL}$, estão discreta mas significantemente elevados quando comparados aos das controles obesas, o que poderia conferir certo grau de proteção contra a doença cardiovascular (24). As pacientes obesas com SOP apresentam maiores níveis de pressão arterial sistólica quando comparadas às magras com SOP e às controles, sugerindo que a hipertensão arterial sustentada seja uma seqüela tardia dos efeitos estimulatórios da hiperinsulinemia sobre o sistema nervoso simpático e o músculo liso vascular (25).

Recentemente, Erhmann e cols. (26) avaliaram 368 pacientes não-diabéticas com SOP, sendo que $80 \%$ apresentavam aumento da relação cintura-quadril (> $88 \mathrm{~cm}), 66 \%$ diminuição dos níveis de HDL-colesterol $(<50 \mathrm{mg} / \mathrm{dL}), 32 \%$ aumento dos níveis de triglicérides ( $\geq 150 \mathrm{mg} / \mathrm{dL}), 21 \%$ hipertensão arterial ( $\geq 130 / 85 \mathrm{mmHg}$ ) e $5 \%$ aumento da glicemia de jejum ( $\geq 110 \mathrm{mg} / \mathrm{dL})$.

A prevalência de síndrome metabólica ( $\mathrm{SM}$, segundo critérios do NCEP ATPIII) nas pacientes com SOP varia de 33 a 43\%, cerca de duas vezes maior que a observada em mulheres da população geral, pareadas para idade e IMC. Nas pacientes com SOP, a SM é diagnosticada usualmente antes do final da terceira década de vida (23\% das pacientes com menos de 19 anos, $45 \%$ daquelas entre 20 e 29 anos e $53 \%$ daquelas entre 30 e 39 anos, versus $0 \%, 6 \%$ e 15\% das controles, respectivamente), com prevalência que se aproxima da observada em mulheres sem SOP entre 50 e 60 anos de idade $(26,27)$ (figura 1 ).

Os valores preditivos positivos para SM das anormalidades da circunferência abdominal, níveis de HDL-colesterol, triglicérides, pressão arterial e glicemia de jejum são, respectivamente, de $41 \%, 48 \%$, 


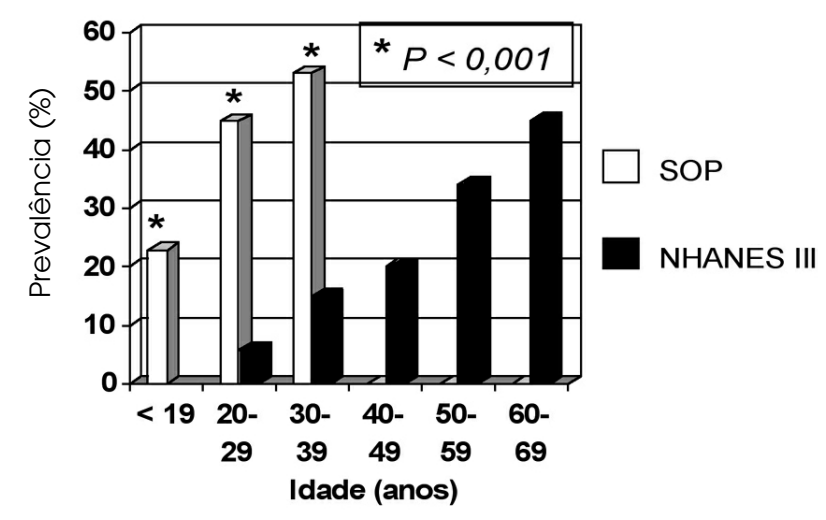

Figura 1. Prevalência de síndrome metabólica (de acordo com critérios do NCEP-ATP III) em pacientes com SOP segundo a faixa etária. (Modificado da ref. 27)

NHANES III: Third National Health and Nutrition Examination Survey (controles)

NCEP-ATP III: National Cholesterol Education Program Adult Treatment Panel III

$83 \%, 78 \%$ e $84 \%$. Os valores preditivos negativos para esses mesmos critérios são, respectivamente, de 96\%, 94\%, 90\%, 79\% e 70\% (26). Embora os níveis de insulina per se não sejam utilizados para o diagnóstico da SOP ou da SM, é amplamente conhecido que a resistência à insulina e a hiperinsulinemia compensatória são fatores importantes na patogênese de ambas as desordens. Nas pacientes adultas com SOP, a prevalência de SM é maior naquelas com IMC superior a $27 \mathrm{~kg} / \mathrm{m}^{2}$ e naquelas com elevados níveis de insulina, mas não se correlaciona com os níveis de andrógenos (26). Diferentemente, nas adolescentes com SOP, a hiperandrogenemia é considerada fator de risco para SM, independentemente da obesidade, do grau de resistência à insulina e dos níveis de SHBG (28).

A presença de SM em mulheres jovens com SOP faz com que elas apresentem risco sete vezes maior de desenvolver doença cardiovascular (27).

\section{SOP E MARCADORES DE ATEROSCLEROSE SUBCLIINICA}

A resistência à insulina está diretamente relacionada à formação da placa aterosclerótica. Ela desempenha papel central no desenvolvimento da disfunção endotelial (evento precoce no processo de aterosclerose), através da indução de distúrbios nas vias de sinalização comuns tanto à ação da insulina como à produção do óxido nítrico, além de aumentar o estresse oxidativo, os níveis de endotelina-1 (ET-1), a atividade do sistema renina-angiotensina e a secreção de hormônios e citocinas pelo tecido adiposo. A disfunção endotelial se caracteriza pela menor produção de óxido nítrico, o qual não só modula o tônus do músculo liso vascular como também inibe vários processos aterogênicos, tais como a adesão de plaquetas e monócitos, a oxidação do LDL, a síntese de citocinas inflamatórias e a proliferação das células musculares lisas vasculares $(29,30)$.

Diminuição da dilatação mediada por fluxo da artéria braquial, cuja resposta é hiperemia da mão, tem sido observada em mulheres jovens com SOP, de forma independente da presença de obesidade $(31,32)$. Essa disfunção endotelial demonstrada na artéria braquial reflete aquela também presente na circulação coronária e é um preditor independente de evento cardiovascular. Correlaciona-se significantemente com a resistência à insulina, os níveis de testosterona total e de colesterol total (31). Além de associar-se a alteração da vasodilatação dependente do endotélio, a resistência à insulina - presente em pacientes com SOP - contribui para diminuição da complascência arterial (hiperplasia do músculo liso vascular, com maior velocidade da onda de pulso na artéria braquial) e ambas as alterações precedem a instalação da hipertensão arterial (33).

As concentrações de proteína $\mathrm{C}$ reativa (PCR), marcador sérico de inflamação crônica de baixo grau e de risco cardiovascular, estão aumentadas tanto nas pacientes com SOP obesas como nas não-obesas, quando comparadas às controles pareadas para o peso $(34,35)$. Níveis de PCR acima de $5 \mathrm{mg} / \mathrm{L}$ (indicativos de alto risco cardiovascular) são observados em $37 \%$ das pacientes com SOP e em só $10 \%$ das controles (36). A PCR também pode estar diretamente envolvida no processo aterogênico (promoção da disfunção endotelial, aumento da síntese de moléculas de adesão solúveis e da secreção de proteínas quimiotáxicas dos monócitos) (34-36). Quarenta e cinco por cento das pacientes com SOP clássica (anovulatória) e 38\% daquelas com SOP ovulatória apresentam dislipidemia ou aumento de PCR ou aumento de homocisteína, diferentemente das mulheres com hirsutismo idiopático e das controles, nas quais a prevalência é de 10\% (37). Diminuição da atividade fibrinolítica (aumento dos níveis de PAI-1) também tem sido relatada na SOP (38).

Em adição aos marcadores séricos, outros indicadores de aterosclerose subclínica têm sido estudados nas pacientes com SOP. Trinta e nove por cento das obesas com SOP apresentam calcificação da artéria coronária na tomografia computadorizada, comparados a $21 \%$ das controles obesas (39). A espessura ínti- 
ma-média da artéria carótida, marcador estrutural de doença arterial que se altera mais tardiamente na progressão da aterosclerose que os marcadores funcionais (dilatação da artéria braquial mediada por fluxo e velocidade da onda de pulso na artéria braquial), também está aumentada nas pacientes com SOP, principalmente após a menopausa, e se correlaciona inversamente com os níveis de SDHEA (40-42).

Apesar de estarem relacionados ao aumento dos níveis de LDL-colesterol e de agirem na vasculatura (aumento da aderência de monócitos, formação de células espumosas, proliferação e migração das células musculares lisas vasculares, aumento da expressão de moléculas de adesão celular e aumento da apoptose das células endoteliais), os andrógenos se contrapõem aos efeitos deletérios da hiperinsulinemia sobre a espessura íntima-média e melhoram a função endotelial indiretamente, por meio da sua conversão local em estradiol, com conseqüente vasodilatação (4l-43).

Recentemente, um estudo mostrou que mesmo jovens não-obesas, não-hipertensas e não-dislipidêmicas com SOP apresentam maiores níveis de insulina e de ETl e maior espessura íntima-média da artéria carótida quando comparadas a mulheres controles, pareadas para idade e IMC, sugerindo que o aumento da espessura íntima-média não está associado exclusivamente à exposição prolongada a perfil cardiovascular adverso (44).

Alterações assintomáticas da função cardíaca (hipertrofia do ventrículo esquerdo e disfunção diastólica) também têm sido observadas em jovens com SOP, muito antes do aparecimento da hipertensão ou do aumento da espessura íntima-média da carótida, em decorrência dos efeitos mitogênicos da insulina $(45,46)$.

\section{SOP, RISCO CARDIOVASCULAR E EVENTOS CARDIOVASCULARES}

$\mathrm{O}$ risco relativo calculado para doença coronariana associado à SOP é de 7,4 (47). A avaliação de mulheres com menos de 60 anos de idade, submetidas à cineangiocoronariografia, revelou que $42 \%$ delas apresentavam morfologia policística dos ovários à US, além de doença arterial coronária mais extensa, com maior número de segmentos com estenose superior a $50 \%$, quando comparadas às mulheres com ovários normais (48). Da mesma forma, mulheres com ciclos menstruais muito irregulares apresentam aumento do risco de desenvolver doença coronariana, mesmo após ajuste para idade, IMC e antecedente familiar de infarto agudo do miocárdio (49).
Diferentemente, Pierpoint e cols. (50) não observaram taxas significantemente maiores de mortalidade relacionada à doença coronariana em 786 pacientes com SOP, avaliadas 30 anos após o diagnóstico, quando comparada às taxas de mortalidade esperadas para o Reino Unido. Wild e cols. (51) relataram que a prevalência de doença coronariana entre 319 pacientes com SOP (média de idade 57 anos), avaliadas 31 anos após o diagnóstico, não foi significantemente maior que a observada em 1.060 controles pareadas para idade $(4,7 \%$ versus $4 \%)$, mas houve aumento significante da ocorrência de doença cérebrovascular, assim como de DM2, o qual abole o efeito protetor do sexo feminino sobre o risco cardiovascular em mulheres na pré-menopausa.

De acordo com o exposto, as evidências sugerindo maior incidência de doença coronariana prematura na SOP são conflitantes. Isso se deve provavelmente à heterogeneidade do critério diagnóstico utilizado nos diversos estudos, ao tamanho das amostras, ao curto prazo de seguimento (há longo período de latência entre o início da aterosclerose e o primeiro evento cardiovascular) e ao estudo de população em idade reprodutiva. Conclui-se, portanto, que embora a SOP possa afetar adversamente o perfil cardiovascular, com desenvolvimento de aterosclerose subclínica, os dados sugestivos da maior incidência de eventos ou mortalidade associada à doença cardiovascular nas pacientes com SOP em idade reprodutiva ainda são limitados. Doença cardiovascular clínica acontece predominantemente na pós-menopausa $(2,40,41,52)$.

\section{TRATAMENTO DA SOP COM AGENTES SENSIBILIZADORES DA INSULINA}

Os objetivos clássicos do tratamento da SOP são melhorar a fertilidade, diminuir as complicações da gravidez (hiperestimulação ovariana, multiparidade, toxemia, diabetes mellitus gestacional e abortamento), regularizar o ciclo menstrual, combater o hiperandrogenismo e prevenir o carcinoma de endométrio. Entretanto, atualmente, novos objetivos se impõem, visando a diminuir o risco de DM2 e, possivelmente, de doença cardiovascular (1).

Redução de peso deve ser recomendada às pacientes obesas ou com sobrepeso, pois perda de aproximadamente $5 \%$ do peso corpóreo pode restaurar a ovulação (1). Vários medicamentos podem ser utilizados no tratamento da SOP, entre eles os anticoncepcionais orais, os anti-andrógenos, os anti-estrógenos e, mais recentemente, os agentes sensibilizadores da 
ação da insulina (tiazolidinedionas e biguanidas). Estes têm sido empregados para reduzir o nível de hiperinsulinemia e seu impacto negativo sobre a função ovariana e a prevenção a longo prazo de suas conseqüências cardiovasculares (1). Estão substituindo o uso já consagrado dos anticoncepcionais orais, os quais podem piorar a resistência à insulina, induzir intolerância à glicose aumentando o risco de desenvolvimento de DM2, elevar os níveis de triglicérides e aumentar o risco cardiovascular também devido às suas ações sobre a coagulabilidade e a reatividade vascular (53).

Metformina, uma biguanida utilizada para tratar pacientes obesos com DM2, tornou-se o agente mais utilizado no tratamento da SOP. Embora ela possa influenciar diretamente a esteroidogênese ovariana in vitro (inibição do CYPI7 com diminuição da expressão de seu RNA mensageiro), a atenuação da produção androgênica pelas células da teca decorre predominantemente da menor concentração de insulina plasmática secundária à inibição da gliconeogênese hepática $(1,54)$. Além disso, há melhora - ainda que discreta - da pressão arterial, diminuição da adiposidade visceral e dos níveis de triglicérides, IL-6, PAI-1, ET-1, PCR e LDL-colesterol, aumento da massa corporal magra e dos níveis de HDL-colesterol, adiponectina e SHBG (55). Recentemente, observouse que tratamento com metformina foi capaz de melhorar a estrutura e função do endotélio de pacientes com SOP jovens e não-obesas, sugerindo que esse agente poderia ser eficaz para reduzir o risco cardiovascular a longo prazo (29).

A metformina pode restaurar a ciclicidade menstrual e é altamente eficaz na indução da ovulação e no aumento da ocorrência de gestação (56). Além disso, as taxas de diabetes gestacional e de abortamento espontâneo são menores em mulheres que engravidam tomando metformina, devido ao aumento dos níveis circulantes de IGFBP-1 e glicodelina, proteína secretada pelo endométrio, que exerce papel crítico durante a implantação do embrião e a manutenção da gestação (57).

Os efeitos benéficos relacionados à metformina parecem ser independentes da diminuição do peso, embora controvérsias ainda existam (1). Mesmo mulheres magras, com índices de ação da insulina normais, respondem ao tratamento, enquanto que pacientes com obesidade grave podem não responder de forma adequada (1). A diminuição do peso de pacientes tratadas com metformina é tanto maior quanto maior o grau de obesidade e maior a dose diária empregada (obesas mórbidas tratadas com $2550 \mathrm{mg} /$ dia chegam a perder até $4 \%$ do peso corpóreo em oito meses) (58). No entanto, interrupção do tratamento promove retorno rápido às condições pré-tratamento (11).

A metformina é classificada como um fármaco de categoria B e parece ser segura durante a gestação tanto para a mãe como para o feto. Efeitos colaterais, tais como náusea, vômito, diarréia e, raramente, acidose láctica, podem limitar seu uso. Está contra-indicada em mulheres com creatinina sérica maior ou igual a $1,4 \mathrm{mg} / \mathrm{dL}$, disfunção hepática e alcoolismo (1).

As tiazolidinedionas (TZDs) pertencem a uma classe mais recente de agentes anti-diabetogênicos orais, que exercem sua ação sensibilizadora à insulina por serem ligantes seletivos do fator de transcrição nuclear PPAR $\gamma$ (receptores nucleares ativados pelo proliferador do peroxissomo $\gamma$ ), presentes no músculo esquelético e tecido adiposo, onde exercem papel central no controle da expressão gênica do adipócito e sua diferenciação (59). As TZDs causam redistribuição da gordura do tecido adiposo visceral para o subcutâneo, com efeitos benéficos sobre fatores endócrinos e metabólicos $(1,59)$.

As TZDs aumentam a sensibilidade à insulina por meio da ativação de múltiplos genes, incluindo o aumento dos transportadores de glicose (GLUT4), e estimulam o estoque de ácidos graxos livres no tecido adiposo, poupando o fígado, o músculo esquelético e, provavelmente, as células $\beta$ das ilhotas pancreáticas da lipotoxicidade. Além disso, aumentam os níveis de adiponectina e HDL-colesterol, exercendo ação antiinflamatória (diminuição dos níveis de IL-6), anti-fibrinolítica (diminuição dos níveis de PAI-1) e vasodilatadora (diminuição dos níveis de ET-1). Na parede vascular, diminuem a produção das moléculas de adesão, proteínas quimiotáxicas dos neutrófilos e metaloproteinase matricial 9, além de aumentar o efluxo do colesterol $(1,59)$.

Os efeitos das TZDs sobre a função ovariana podem ser indiretos (devidos à ação sistêmica sensibilizadora da insulina e redução da hiperinsulinemia), tais como a diminuição dos níveis de testosterona total e livre e aumento dos níveis de SHBG e de IGFBP-1. No entanto, também têm sido descritos efeitos diretos, os quais podem ser independentes da insulina (aumento dos níveis de progesterona e de IGFBP-1 e diminuição dos níveis de testosterona total e de estradiol), ou decorrentes do aumento local do efeito da insulina (diminuição da produção de IGFBP-1 e aumento da produção de estradiol, na presença de altas concentrações de insulina) (60).

A troglitazona, um membro da família das TZDs, mostrou exercer efeitos benéficos (de maneira 
dose-dependente) sobre a função ovulatória e o hiperandrogenismo de mulheres com SOP, mesmo na ausência de hiperinsulinemia marcante, mas tal agente foi retirado do mercado devido à hepatotoxicidade (1). Em doses farmacológicas (11-12 $\mu \mathrm{M}$ ), a troglitazona inibe diretamente a biossíntese androgênica, através da inibição da $3 \beta$-hidroxiesteróide desidroge-

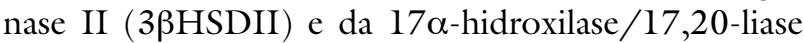
(diminuição da transcrição do gene CYPI7 e da fosforilação da 17,20-liase). A rosiglitazona também é capaz de inibir a $17 \alpha$-hidroxilase $/ 17,20$-liase e a $3 \beta$ HSDII, porém em concentrações supra-farmacológicas $(86-90 \mu \mathrm{M})$ e a pioglitazona apresenta mínimos efeitos inibitórios (somente em concentrações superiores a $100 \mu \mathrm{M})(61,62)$.

Regiões diferentes das TZDs estão envolvidas com a ligação ao PPAR $\gamma$ e a inibição enzimática. Esta não se correlaciona com a afinidade de ligação ao PPAR $\gamma$, uma vez que tanto a rosiglitazona como a pioglitazona ligam-se com maior afinidade ao PPAR $\gamma$ e são sensibilizadores mais potentes da insulina que a troglitazona $(61,62)$.

A administração de rosiglitazona $(4 \mathrm{mg} / \mathrm{dia})$ ou de pioglitazona $(30 \mathrm{mg} / \mathrm{dia})$ a mulheres obesas e nãoobesas com SOP levou à melhora da resistência à insulina, diminuição da produção androgênica ovariana, independente da alteração dos níveis de LH, restauração da ovulação espontânea e diminuição dos níveis circulantes de SDHEA $(63,64)$. Romualdi e cols. (65) avaliaram os efeitos da pioglitazona (45 $\mathrm{mg} /$ dia, durante 6 meses) em pacientes com SOP normo e hiperinsulinêmicas e relataram melhora significante do hirsutismo e restauração da ciclicidade menstrual nos dois grupos de pacientes, sugerindo que a hiperinsulinemia não constitui um determinante da resposta clínica às TZDs.

Recentemente, um estudo comparou as respostas dos andrógenos séricos e da resistência insulínica após a administração, durante seis meses, de metformina $(2550 \mathrm{mg} /$ dia $)$ ou pioglitazona (30 $\mathrm{mg} /$ dia), em mulheres obesas com SOP. O peso corpóreo aumentou significantemente após o uso da pioglitazona, mas ambos os agentes diminuíram igualmente o grau de hirsutismo e as concentrações séricas de testosterona livre e de androstenediona. A insulinemia de jejum diminuiu nos dois grupos, mas a diminuição da área sob a curva da insulina durante o teste oral de tolerância à glicose foi significantemente maior no grupo tratado com pioglitazona (66).

Tanto a rosiglitazona como a pioglitazona são classificadas como fármacos de categoria $\mathrm{C}$, uma vez que estudos em animais demonstraram retardo do cresci- mento intra-uterino. Portanto, as mulheres tratadas com esses medicamentos devem receber aconselhamento contraceptivo e ser instruídas a interromper o tratamento tão $\operatorname{logo}$ a gestação seja confirmada. A pioglitazona, por ser agonista do PPAR $\gamma$ e agonista parcial do PPAR $\alpha$, diminui também os níveis de triglicérides, diferentemente da rosiglitazona, que é um agonista puro do PPAR . Os principais efeitos colaterais observados com estas TZDs são: aumento de peso, retenção de fluido, edema periférico e discreta diminuição do hematócrito. Não há relatos de hepatotoxicidade (1).

\section{CONCLUSÃO}

A SOP representa o maior grupo de mulheres jovens de alto risco para o possível desenvolvimento de doença cardiovascular, a qual pode ser diagnosticada muitos anos antes do início dos sintomas. A resistência à insulina é o elo entre a SOP e a SM, também condição de risco cardiovascular aumentado. Medidas cardioprotetoras devem ser adotadas para melhorar a função endotelial destas pacientes, incluindo desde dieta, prática de exercícios físicos e interrupção do tabagismo, até o controle da pressão arterial, o uso de baixas doses de aspirina, estatinas e, principalmente, agentes sensibilizadores da ação da insulina.

\section{REFERÊNCIAS}

1. Ehrmann DA. Polycystic ovary syndrome. $\mathbf{N}$ Engl J Med 2005;352:1223-36.

2. Azziz R, Marin C, Hoq L, Badamgarav E, Song P. Health care-related economic burden of the polycystic ovary syndrome during the reproductive life span. J Clin Endocrinol Metab 2005;90:4650-8.

3. Carmina E, Rosato F, Jannì A, Rizzo M, Longo RA. Relative prevalence of different androgen excess disorders in 950 women referred because of clinical hyperandrogenism. J Clin Endocrinol Metab 2006;91:2-6.

4. Azziz R, Sanchez A, Knochenhauer ES, Moran C, Lazenby J, Stephens KC, et al. Androgen excess in women: experience with over 1,000 consecutive patients. J Clin Endocrinol Metab 2004;89:453-62.

5. The Rotterdam ESHRE/ASRM-sponsored PCOS consensus workshop group. Revised 2003 consensus on diagnostic criteria and long-term health risks related to polycystic ovary syndrome (PCOS). Hum Reprod 2004; 19:41-7.

6. Escobar-Morreale HF, Luque-Ramírez M, San Millán JL. The molecular-genetic basis of functional hyperandrogenism and the polycystic ovary syndrome. Endocr Rev 2005;26:251-82.

7. Wickenheisser JK, Nelson-DeGrave VL, Hendricks KL, Legro RS, Strauss JF, McAllister M. Retinoids and retinol 
differentially regulate steroid biosynthesis in ovarian theca cells isolated from normal cycling women and women with polycystic ovary syndrome. J Clin Endocrinol Metab 2005;90:4858-65.

8. Wickenheisser JK, Nelson DeGrave VL, McAllister JM. Dysregulation of cytochrome P450 17 $\alpha$-hydroxylase messenger ribonucleic acid stability in theca cells isolated from women with polycystic ovary syndrome. J Clin Endocrinol Metab 2005;90:1720-7.

9. Qin K, Ehrmann DA, Cox N, Refetoff S, Rosenfield RL. Identification of a functional polymorphism of the human type $517 \beta$-hydroxysteroid dehydrogenase gene associated with polycystic ovary syndrome. J Clin Endocrinol Metab 2006;91:270-6.

10. San Millán JL, Botella-Carretero Jl, Álvarez-Blasco F, Luque-Ramírez M, Sancho J, Moghetti P, et al. A study of the hexose-6-phosphate dehydrogenase gene R453Q and $11 \beta$-hydroxysteroid dehydrogenase type 1 gene 83557insA polymorphism in the polycystic ovary syndrome. J Clin Endocrinol Metab 2005;90:4157-62.

11. Ibánez L, Valls C, Marcos MV, Ong K, Dunger DB, de Zegher F. Insulin sensitization for girls with precocious pubarche and with risk for polycystic ovary syndrome: effects of prepubertal initiation and post pubertal discontinuation of metformin treatment. J Clin Endocrino Metab 2004:89:4331-7.

12. Dunaif A. Insulin resistance and the polycystic ovary syndrome: mechanism and implications for pathogenesis. Endocr Rev 1997; 18:774-800.

13. Tsilchorozidou T, Honou JW, Conway GS. Altered cortisol metabolism in polycystic ovary syndrome: insulin enhances $5 \alpha$-reduction but not the elevated adrenal steroid production rates. J Clin Endocrinol Metab 2003:88:5907-13

14. Baillargeon JP, Nestler JE. Polycystic ovary syndrome: a syndrome of ovarian hypersensitivity of insulin? J Clin Endocrinol Metab 2006;91:22-4.

15. Dunaif A, Segal KR, Shelley DR, Green G, Dobrjansky A, Licholai T. Evidence for distinctive and intrinsic defects in insulin action in polycystic ovary syndrome. Diabetes 1992;41:1257-66

16. Puder JJ, Varga S, Kraenzlin M, de Geyter C, Keller U, Müller B. Central fat excess in polycystic ovary syndrome: relation to low-grade inflammation and insulin resistance. J Clin Endocrinol Metab 2005:90:6014-21.

17. Fernández-Real JM, Ricart W. Insulin resistance and chronic cardiovascular inflammatory syndrome. Endocr Rev 2003:24:278-301

18. Miller WL, Nestler JE, Futterweit W. Three viewpoints on polycystic ovary syndrome. Endocr News 2004;29:12-9.

19. González F, Minium J, Rote NS, Kirwan JP. Hyperglycemia alters tumor necrosis factor-a release from mononuclear cells in women with polycystic ovary syndrome. J Clin Endocrinol Metab 2005;90:5336-42.

20. González F, Rote NS, Minium J, Kirwan JP. Reactive oxygen species-induced oxidative stress in the development of insulin resistance and hyperandrogenism in polycystic ovary syndrome. J Clin Endocrinol Metab 2006;91:336-40.
21. Legro RS, Kunselman AR, Dodson WC, Dunaif A. Prevalence and predictors of risk for type 2 diabetes mellitus and impaired glucose tolerance in polycystic ovary syndrome: a prospective, controlled study in 254 affected women. J Clin Endocrinol Metab 2005;84:165-9.

22. Ehrmann DA, Kasza K, Azziz R, Legro RS, Ghazzi MN; The PCOS/Troglitazone Study Group. Effects of race and family history of type 2 diabetes on metabolic status of women with polycystic ovary syndrome. J Clin Endocrinol Metab 2005;90:66-71.

23. Legro RS, Gnatuk CL, Kunselman AR, Dunaif A. Changes in glucose tolerance over time in women with polycystic ovary syndrome: a controlled study. J Clin Endocrinol Metab 2005:90:3236-42.

24. Legro RS, Kunselman AR, Dunaif A. Prevalence and predictors of dyslipidemia in women with polycystic ovary syndrome. Am J Med 2001;1 11:665-6.

25. Conway GS, Agrawal R, Betteridge DJ, Jacobs HS. Risk factors for coronary artery disease in lean and obese women with polycystic ovary syndrome. Clin Endocrinol (Oxf) 1992:37:119-25.

26. Ehrmann DA, Liljenquist DR, Kasza K, Azziz R, Legro RS, Ghazzi MN, et al. Prevalence and predictors of the metabolic syndrome in women with polycystic ovary syndrome (PCOS). J Clin Endocrinol Metab 2006:91:48-53.

27. Apridonidze T, Essah PA, luorno MJ, Nestler JE. Prevalence and characteristics of the metabolic syndrome in women with polycystic ovary syndrome. J Clin Endocrinol Metab 2005;90:1929-35.

28. Coviello AD, Legro RS, Dunaif $A$. Adolescent girls with polycystic ovary syndrome have an increased risk of the metabolic syndrome associated with increasing androgen levels independent of obesity and insulin resistance. J Clin Endocrinol Metab 2005; (Epub ahead of print)

29. Orio Jr F, Palomba S, Cascella T, de Simone B, Manguso $\mathrm{F}$, Savastano S, et al. Improvement in endothelial structure and function after metformin treatment in young normal-weight women with polycystic ovary syndrome: results of a 6-month study. J Clin Endocrinol Metab 2005:90:6072-6.

30. Kadowaki T, Yamauchi T. Adiponectin and adiponectin receptors. Endocr Rev 2005;26:439-51.

31. Kravariti M, Naka KK, Kalantaridou SN, Kazakos N, Katsouras CS, Makrigiannakis A, et al. Predictors of endothelial dysfunction in young women with polycystic ovary syndrome. J Clin Endocrinol Metab 2005;90:508895.

32. Meyer C, Mcgrath BP, Teede HJ. Overweight women with polycystic ovary syndrome have evidence of subclinical cardiovascular disease. J Clin Endocrinol Metab 2005:90:5711-6.

33. Kelly CJG, Speirs A, Gould GW, Petrie JR, Lyall H, Connell JMC. Altered vascular function in young women with polycystic ovary syndrome. J Clin Endocrinol Metab 2002;87:742-6.

34. Kelly CCJ, Lyall H, Petrie JR, Gould GW, Connell JMC, Sattar N. Low grade chronic inflammation in women with polycystic ovarian syndrome. J Clin Endocrinol Metab 2001:86:2453-5. 
35. Tarkun I, Arslan BÇ, Cantürk Z, Türemen E, Sahin T, Duman C. Endothelial dysfunction in young women with polycystic ovary syndrome: relationship with insulin resistance and low-grade chronic inflammation. J Clin Endocrinol Metab 2004;89:5592-6.

36. Boulman N, Levy Y, Leiba R, Shachar S, Linn R, Zinder O, et al. Increased $C$-reactive protein levels in the polycystic ovary syndrome: a marker of cardiovascular disease. J Clin Endocrinol Metab 2004;89:2160-5.

37. Carmina E, Chu MC, Longo RA, Rini GB, Lobo RA. Phenotypic variation in hyperandrogenic women influences the findings of abnormal metabolic and cardiovascular risk parameters. J Clin Endocrinol Metab 2005:90:2545-9.

38. Ehrmann DA, Schneider DJ, Sobel BE, Cavaghan MK, Imperial J, Rosenfield RL, et al. Troglitazone improves defects in insulin action, insulin secretion, ovarian steroidogenesis, and fibrinolysis in women with polycystic ovary syndrome. J Clin Endocrinol Metab 1997:82:2108-16.

39. Christian RC, Dumesic DA, Behrenbeck T, Oberg AL, Sheedy II PF, Fitzpatrick LA. Prevalence and predictors of coronary artery calcification in women with polycystic ovary syndrome. J Clin Endocrinol Metab 2003;88:2562-8.

40. Legro RS. Polycystic ovary syndrome and cardiovascular disease: a premature association? Endocr Rev 2003;24:302-12.

41. Meyer C, McGrath BP, Cameron J, Kotsopoulos D, Teede HJ. Vascular dysfunction and metabolic parameters in polycystic ovary syndrome. J Clin Endocrinol Metab 2005;90:4630-5.

42. Vryonidou A, Papatheodorou A, Tavridou A, Terzi T, Loi V, Vatalas IA, et al. Association of hyperandrogenemic and metabolic phenotype with carotid intima-media thickness in young women with polycystic ovary syndrome. J Clin Endocrinol Metab 2005:90:2740-6.

43. Liu PY, Death AK, Handelsman DJ. Androgens and cardiovascular disease. Endocr Rev 2003:24:313-40.

44. Orio Jr F, Palomba S, Cascella T, de Simone B, di Biasi S, Russo T, et al. Early impairment of endothelial structure and function in young normal-weight women with polycystic ovary syndrome. J Clin Endocrinol Metab 2004:89:4588-93.

45. Orio Jr F, Palomba S, Spinelli L, Cascella T, Tauchmanovà $\mathrm{L}$, Zullo $\mathrm{F}$, et al. The cardiovascular risk of young women with polycystic ovary syndrome: an observational, analytical, prospective case-control study. J Clin Endocrinol Metab 2004:89:3696-701.

46. Guzick DS. Editorial: cardiovascular risk in PCOS. J Clin Endocrinol Metab 2004;89:3694-5.

47. Dahlgren E, Janson PO, Johansson S, Lapidus L, Oden A. Polycystic ovary syndrome and risk factor model based on a prospective population study of women. Acta Obstet Gynecol Scand 1992;71:599-604.

48. Birdsall MA, Farquhar CM, White HD. Association between polycystic ovaries and extent of coronary artery disease in women having cardiac catheterization. Ann Intern Med 1997; 126:32-5.

49. Solomon CG, Hu FB, Dunaif A, Rich-Edwards JE, Stampfer MJ, Willett WC, et al. Menstrual cycle irregularity and risk for future cardiovascular disease. J Clin Endocrinol Metab 2002:87:2013-7.
50. Pierpoint T, McKeigue PM, Isaacs AJ, Wild SH, Jacobs HS. Mortality of women with polycystic ovary syndrome at long-term follow-up. J Clin Epidemiol 1998:51:581-6.

51. Wild S, Pierpoint T, McKeigue P, Jacobs H. Cardiovascular disease in women with polycystic ovary syndrome at long-term follow-up: a retrospective cohort study. Clin Endocrinol 2000;52:595-600.

52. Taponen S, Martikainen $H$, Järvelin MR, Sovio U, Laitinen J, Pouta A, et al. Metabolic cardiovascular disease risk factors in women with self-reported symptoms of oligomenorrhea and/or hirsutism: Northern Finland birth cohort 1966 study. J Clin Endocrinol Metab 2004;89:21 14-8.

53. Diamanti-Kandarakis E, Baillargeon JP, luorno MJ, Jakubowicz DJ, Nestler JE. A modern medical quandary: polycystic ovary syndrome, insulin resistance and oral contraceptive pills. J Clin Endocrinol Metab 2003:88:1927-32

54. Attia GR, Rainey WE, Carr BR. Metformin directly inhibits androgen production in human thecal cells. Fertil Steril 2001;76:517-24.

55. Ibánez L, Zegher F. Ethinylestradiol-drospirenone, flutamide-metformin or both for adolescents and women with hyperinsulinemic hyperandrogenism: opposite effects on adipocytokines and body adiposity. J Clin Endocrinol Metab 2004;89:1592-7.

56. Norman RJ. Metformin - comparison with other therapies in ovulation induction in polycystic ovary syndrome. J Clin Endocrinol Metab 2004;89:4797-800.

57. Jakubowicz DJ, Seppälä M, Jacubowicz S, RodriguezArmas $\mathrm{O}$, Rivas-Santiago $\mathrm{A}$, Koistinen $\mathrm{H}$, et al. Insulin reduction with metformin increases luteal phase serum glycodelin and insulin-like growth factor-binding protein 1 concentrations and enhances uterine vascularity and blood flow in the polycystic ovary syndrome. J Clin Endocrinol Metab 2001;86:1126-33.

58. Harborne LR, Sattar N, Norman JE, Fleming R. Metformin and weight loss in obese women with polycystic ovary syndrome: comparison of doses. J Clin Endocrinol Metab 2005:90:4593-8.

59. Yki-Järvinen H. Thiazolidinediones. N Engl J Med 2004; 351:1106-18.

60. Seto-Young D, Paliou M, Schlosser J, Avtanski D, Park A Patel $P$, et al. Direct thiazolidinedione action in the human ovary: insulin-independent and insulin-sensitizing effects on steroidogenesis and insulin-like growth factor binding protein-1 (IGFBP-1) production. J Clin Endocrinol Metab 2005;90:6099-105.

61. Arlt W, Auchus J, Miller WL. Thiazolidinediones but not metfomin directly inhibit the steroidogenic enzymes P450c17 and 33-hydroxysteroid dehydrogenase. J Biol Chemistry 2001;20:16767-71.

62. Arlt W, Neogi P, Gross C, Miller WL. Cinnamic acid based thiazolidinediones inhibit human $\mathrm{P} 450 \mathrm{c} 17$ and $3 \beta$ hydroxysteroid dehydrogenase and improve insulin sensitivity independent of PPARy agonist activity. J Mol Endocrinol 2004;32:425-36.

63. Sepilian V, Nagamani M. Effects of rosiglitazone in obese women with polycystic ovary syndrome and severe insulin resistance. J Clin Endocrinol Metab 2005:90:60-5. 
64. Brettenthaler N, De Geyter C, Huber PR, Séller U. Effects of the insulin sensitizer pioglitazone on insulin resistance, hyperandrogenism and ovulatory dysfunction in women with polycystic ovary syndrome. J Clin Endocrinol Metab 2004:89:3835-40

65. Romualdi D, Guido M, Ciampelli M, Giuliani M, Leoni F, Perri $C$, et al. Selective effects of pioglitazone on insulin and androgen abnormalities in normo- and hyperinsulinaemic obese patients with polycystic ovary syndrome. Hum Reprod 2003; 18:1210-8.

66. Ortega-González C, Luna S, Hernández L, Crespo G, Aguayo $P$, Artega-Troncoso $G$, et al. Responses of serum androgen and insulin resistance to metformin and pioglitazone in obese, insulin-resistant women with polycystic ovary syndrome. J Clin Endocrinol Metab 2005:90:1360-5.

\section{Endereço para correspondência:}

Regina do Carmo Silva Disciplina de Endocrinologia

Departamento de Medicina

Universidade Federal de São Paulo (UNIFESP)

Rua Botucatu 740

04034-970 São Paulo, SP

E-mail: rcarmo@osite.com.br 